\title{
Prevalência de ansiedade e fatores associados em adultos
}

\author{
Prevalence of anxiety and associated factors in adults \\ Camilla Oleiro da Costa ${ }^{1}$ \\ https://orcid.org/0000-0003-2476-7153 \\ Jerônimo Costa Branco² \\ https://orcid.org/0000-0001-6750-2740 \\ Igor Soares Vieira ${ }^{3}$ \\ https://orcid.org/0000-0002-3263-8397 \\ Luciano Dias de Mattos Souza ${ }^{3}$ \\ https://orcid.org/0000-0001-9965-4837 \\ Ricardo Azevedo da Silva ${ }^{3}$ \\ https://orcid.org/0000-0002-9114-7037
}

\section{RESUMO}

Objetivo: Identificar a prevalência de transtornos de ansiedade em uma amostra de base populacional e fatores associados. Métodos: Estudo transversal de base populacional realizado com indivíduos entre 18 e 35 anos. As variáveis sociodemográficas, índice de massa corporal, presença de doença crônica, abuso de álcool e tabagismo foram analisadas. Os transtornos de ansiedade foram verificados pela Mini Internacional Neuropsychiatric Interview 5.0. Foi utilizado o teste Qui-quadrado, considerando o intervalo de confiança de 95\%. Resultados: A amostra foi constituída por 1.953 pessoas. A prevalência de transtornos de ansiedade foi de $27,4 \%$. Agorafobia $(17,9 \%)$ e transtorno de ansiedade generalizada $(14,3 \%)$ foram os quadros mais prevalentes. Mulheres apresentaram maior prevalência de ansiedade, com 32,5\%, quando comparadas aos homens $(21,3 \%)(p<0,001)$. As variáveis sexo, anos de estudo, renda, doença crônica, tabagismo e álcool foram associadas a mais de três transtornos de ansiedade investigados $(p<0,001)$. Conclusão: Os dados demonstram que os transtornos de ansiedade são muito frequentes em adultos, sendo mais prevalentes entre as mulheres. Estão associa-

\section{Palavras-chave}

Prevalência, transtornos de ansiedade, adulto jovem. das aos transtornos ansiosos, principalmente, as condições socioeconômicas e substâncias licitas. Conhecer as prevalências dos transtornos de ansiedade e fatores associados pode auxiliar profissionais de saúde a elaborarem melhores diagnósticos e tratamentos.

\section{ABSTRACT}

Objective: Identify the prevalence of anxiety disorders in a population sample and associated factors. Methods: A cross-sectional study of population base conducted with individuals between 18 and 35 years old. Sociodemographic variables, body mass index (BMI), presence of diseases, and use of alcohol and smoke were analyzed. The anxiety disorders were verified by the clinical interview Mini International Neuropsychiatric Interview 5.0. For the analysis, was

1 Universidade Federal de Pelotas, Pelotas, RS, Brasil 2 Universidade Franciscana, Santa Maria, RS, Brasil 


\section{Keywords}

Prevalence, anxiety disorders, young adult. used the Chi-square test, considering a confidence interval of 95\%. Results: The prevalence of general anxiety was 27.4\%. Agoraphobia (17.9\%) and generalized anxiety disorder (14.3\%) were the most prevalent. Women presented a higher prevalence of general anxiety with $32.5 \%$ when compared with man $(21,3 \%)$ ( $p<0.001$ ). The variables gender, years of study, income, health problems, smoking, and alcohol were associated to more than three anxiety disorders investigated ( $p<0,001$ ). Conclusions: The findings demonstrate that anxiety disorders are frequent in adults, being more prevalent among women. It was associated mainly with socioeconomic conditions and legal substances. Knowing the prevalence of anxiety disorders and associated factors can help health professionals to develop better diagnoses and treatments.

\section{INTRODUÇÃO}

Mais de 450 milhões de pessoas sofrem com algum transtorno de saúde mental' ${ }^{1}$. No ano de 2013, a revisão com 87 estudos feitos por Baxter et al., em 44 países, estimou a prevalência atual dos transtornos de ansiedade em 7,3\% (4,8\%-10,9\%)2. Os transtornos de ansiedade diferem entre si nos objetos ou situações que induzem o medo, ansiedade ou comportamento de esquiva ${ }^{3}$. São quadros clínicos cujos sintomas de ansiedade são primários (não são derivados de outras doenças psiquiátricas como depressão ou psicoses, por exemplo)4

Segundo a classificação do Manual Diagnóstico e Estatístico de Transtornos Mentais (DSM-IV), agorafobia, transtorno de pânico, transtorno obsessivo-compulsivo (TOC), fobia social, transtorno de estresse pós-traumático (TEPT) e transtorno de ansiedade generalizada (TAG) são alguns dos transtornos de ansiedade 3 .

As comorbidades são frequentes nos transtornos de ansiedade, variando entre outros transtornos psiquiátricos até doenças cardiovasculares e renais 5 . Os transtornos de ansiedade geralmente prejudicam a vida diária dos indivíduos, pois muitos deixam de realizar atividades rotineiras por medo das crises ou sintomas. As situações que provocam ansiedade algumas vezes são suportadas com grande sofrimento e muitas das atividades exigem a participação de outras pessoas para que sejam realizadas - o que pode afetar a qualidade de vida e diminuir o grau de independência ${ }^{3,5}$. Rompimentos sociais e de relacionamentos e abandono de atividades consideradas prazerosas também podem acontecer. Dessa forma, a identificação desses acontecimentos pode direcionar ao tratamento precoce, diminuindo a gravidade desses quadros ao longo do desenvolvimento da doença.

Assim, tendo em vista a alta a prevalência dos transtornos de ansiedade, prejuízos que esses quadros podem gerar na vida dos indivíduos e poucos estudos existentes na literatura sobre transtornos ansiosos, fazem-se necessárias a investigações sobre o tema e a diferenciação das taxas de prevalências de diferentes quadros de transtornos de ansiedade para a condução de tratamentos adequados. Entender as interferências dos quadros de saúde mental no cotidiano das pessoas e como esses fatores se associam pode contribuir para o desenvolvimento de políticas de saúde e a melhoria dos serviços de atendimento.

O Relatório Mundial de Saúde recomenda que estudos direcionados para o desenvolvimento e a criação de políticas e serviços sejam uma das cinco prioridades para o campo da saúde mental ${ }^{6}$. Sendo assim, o objetivo deste estudo foi identificar a prevalência de transtornos de ansiedade em uma amostra de base populacional, bem como seus fatores associados.

\section{MÉTODOS}

Este é um estudo transversal de base populacional, realizado com adultos entre 18 e 35 anos residentes na região sul do Brasil, realizado entre os anos de 2011 e 2014. A seleção da amostra foi realizada por conglomerados, considerando a divisão censitária de Pelotas de 2010: a cidade é dividida em 495 setores urbanos pelo Instituto Brasileiro de Geografia e Estatística (IBGE; http://www.ibge.gov.br).

Para garantir a inclusão de uma amostra abrangente e representativa, 83 setores censitários foram sorteados de forma sistemática com o pulo de seis setores. A seleção dos domicílios nos setores sorteados foi realizada de acordo com uma amostragem sistemática: o primeiro domicílio foi o da esquina preestabelecida pelo IBGE como início do setor e o intervalo de seleção determinado por um pulo de quatro domicílios entre os sorteados.

Assim, foram incluídos no estudo os indivíduos sorteados que, após terem conhecimento dos objetivos do estudo, aprovaram a sua participação e assinaram o Termo de Consentimento Livre e Esclarecido. Foram excluídos indivíduos que, por algum motivo físico ou cognitivo, não foram capazes de compreender e responder aos instrumentos propostos no estudo. Este estudo foi aprovado pelo comitê de ética em pesquisa da Universidade Católica de Pelotas (UCPel), por meio do protocolo no 15/2010.

Alunos do Centro de Ciência da Vida e da Saúde da UCPel, previamente treinados e identificados, dirigiram-se às residências sorteadas para a realização da entrevista. Na ocasião das entrevistas domiciliares, foram coletados dados sociodemográficos, antropométricos e sobre abuso de ál- 
cool e doenças crônicas, e foi aplicada uma entrevista clínica diagnóstica.

Os dados sociodemográficos foram obtidos por meio de um questionário constituído pelas variáveis: sexo, idade, cor da pele, avaliação econômica, trabalho e situação conjugal. A avaliação econômica dos participantes foi realizada pelo Critério do IEN (Índice Econômico Nacional). A avaliação se baseia no acúmulo de bens materiais e na escolaridade do chefe da família. Esse critério gera uma variável contínua, que foi apresentada por tercis. O uso de tabaco foi questionado por meio da pergunta: "Você faz uso de cigarro?". O abuso do álcool foi avaliado por meio do Cut down, Annoyed, Guilty, Eye-opener (CAGE) (sensibilidade $\geq 0,88$, especificidade $\geq 0,81)^{7}$. Esse instrumento contém quatro questões, e a resposta afirmativa em duas ou mais é um indicativo de diagnóstico de abuso de álcool. Já o uso de tabaco foi mensurado pela seguinte pergunta: "Você fez uso de pelo menos um cigarro/dia na última semana?".

Para determinar o índice de massa corporal (IMC), a altura foi mensurada com uma fita métrica e o peso com balança de bioimpedância Tanita ${ }^{\circ}$ BC-554. O IMC foi dividido em três grupos (peso normal, sobrepeso e obeso), em uma classificação baseada nas Diretrizes Brasileiras de Obesidade ${ }^{8}$. Como peso normal, foi considerado o IMC entre 18,5 e 24,9, sobrepeso entre 25 e 29,9 e obeso a partir de 30.

O transtorno de ansiedade foi investigado por meio da entrevista clínica estruturada Mini International Neuropsychiatric Interview 5.0 (MINI) $)^{9}$. Esse instrumento é amplamente utilizado na prática clínica e de pesquisa, e os entrevistadores foram previamente treinados por psicólogos responsáveis pelo estudo. Os índices de confiabilidade do MINI são satisfatórios quando comparados a vários critérios de referência (DSM-IV e Classificação Internacional de Doenças - CID-10), em diferentes contextos (unidades psiquiátricas e centros de atenção primária). Ainda de acordo com essa autora, o MINI mostrou qualidades psicométricas similares às de outras entrevistas diagnósticas padronizadas mais complexas, permitindo uma redução de 50\% ou mais no tempo da avaliação.

Para esse estudo, foi considerada para o transtorno de ansiedade atual a presença de alguns dos quadros avaliados pela MINI, seja agorafobia atual (sensibilidade $\geq 0,59$, especificidade $\geq 0,95$ ), transtorno de pânico atual (sensibilidade $\geq$ 0,67 , especificidade $\geq 0,97$ ), TOC (sensibilidade $\geq 0,62$, especificidade $\geq 0,98$ ), fobia social (sensibilidade $\geq 0,72$, especificidade $\geq 0,88$ ), TEPT (sensibilidade $\geq 0,85$, especificidade $\geq 0,96$ ) e ansiedade generalizada (sensibilidade $\geq 0,67$, especificidade $\geq$ $0,92)$, e também avaliamos esses quadros de forma individual.

Para o tratamento estatístico, foi utilizado o programa Statistical Package for the Social Science (SPSS), versão 21.0, no qual se procedeu à análise inicial com o objetivo de obter frequência dos transtornos mentais avaliados, além de caracterizar a amostra do estudo. O teste Qui-quadrado foi utilizado para as análises de inferência, visando descrever a associação entre os transtornos analisados com as variáveis sociodemográficas IMC, doenças crônicas, abuso de álcool e tabaco. Foram consideradas associações estatisticamente significativas quando $p<0,05$.

\section{RESULTADOS}

A amostra foi constituída de 2.361 adultos jovens (265 foram considerados perdas, por não serem reencontrados após a sua identificação e inclusão no estudo para responderem à pesquisa, e 143 recusaram-se a participar do estudo), totalizando um $n$ amostral de 1.953. A amostra foi composta em $54,9 \%(n=1.073)$ por mulheres e em $45,1 \%(n=880)$ por homens. As características gerais da amostra estão descritas na tabela 1, bem como os fatores associados ao transtorno de ansiedade e os dados estratificados por sexo relacionados ao transtorno de ansiedade.

A prevalência de transtorno de ansiedade foi de $27,4 \%$ ( $n=$ 536). Avaliando individualmente os quadros de transtornos ansiosos, as maiores taxas de prevalência foram 17,9\% ( $n=350)$ para agorafobia e 14,3\% ( $n=278)$ para TAG. A fobia social apresentou prevalência de 5,4\% ( $n=105)$, TOC de 4,2\% $(n=82)$, transtorno de pânico de 3,6\% ( $n=71)$ e TEPT de 3,0\% $(n=58)$.

As mulheres tiveram maior prevalência de ansiedade de modo geral, quando comparadas aos homens; o sexo feminino alcançou 32,5\% ( $n=349)$, enquanto o masculino, $21,3 \%$ $(n=187)(p<0,001)$. As variáveis anos de estudo, renda, doença crônica, tabagismo e abuso de álcool estavam associadas a ambos os sexos. $(p \leq 0,05)$ e a cor da pele, apenas ao sexo feminino $(p<0,034)$ (Tabela 1).

Quando os quadros foram investigados de maneira individual, a agorafobia esteve associada ao sexo feminino ( $p<0,001)$, grupo com menos anos de estudo $(p<0,001)$, classificados no menor tercil de renda ( $p<0,001)$, grupo que relatou a presença de doença crônica $(p<0,001)$ e que fazia uso de tabaco $(p<0,001)$ e abuso de álcool $(p<0,001)$. Para o transtorno de pânico, aqueles classificados no menor tercil de renda ( $p<0,032)$ e os fumantes $(p<0,014)$ mostraram associação significativa.

O TOC foi associado ao sexo feminino ( $p=0,009)$, grupo com menos anos de estudo $(p=0,004)$, grupo que relatou a presença de doença crônica ( $p=0,008)$, fumantes $(p<0,001)$ e os que abusavam de álcool $(p=0,002)$. A fobia social esteve associada a sexo feminino ( $p<0,001)$, cor de pele não branca $(p=0,008)$, aqueles no menor tercil de renda $(p<0,001)$, grupo com doença crônica ( $p<0,001)$, aqueles com menos anos de estudo ( $p<0,001)$ e fumantes $(p<0,001)$.

O TEPT esteve associado ao sexo feminino $(p=0,004)$, ao grupo com menos anos de estudo ( $p<0,001)$, menor tercil de renda ( $p<0,001)$, doença crônica $(p=0,002)$, fumantes $(p<0,001)$ e abusadores de álcool $(p=0,002)$. O TAG esteve associado ao sexo feminino $(p<0,001)$, ao grupo com mais 
Tabela 1. Características gerais da amostra, prevalência de transtornos de ansiedade geral e estratificação por sexo de acordo com os fatores associados ao transtorno de ansiedade geral

\begin{tabular}{|c|c|c|c|c|c|c|c|}
\hline \multirow{2}{*}{ Variáveis } & \multirow{2}{*}{$\begin{array}{c}\text { Característica da } \\
\text { amostra }\end{array}$} & \multirow{2}{*}{$\begin{array}{l}\text { Transtorno de } \\
\text { ansiedade }\end{array}$} & \multirow{2}{*}{$p$} & \multicolumn{4}{|c|}{ Transtorno de ansiedade } \\
\hline & & & & Mulheres & $\mathrm{p}$ & Homens & $\mathrm{p}$ \\
\hline Idade & & & 0,564 & & 0,924 & & 0,614 \\
\hline 18 a 23 anos & $762(39,0)$ & $201(26,4)$ & & $126(31,8)$ & & $75(20,5)$ & \\
\hline 24 a 29 anos & $622(31,8)$ & $180(28,9)$ & & $118(33,1)$ & & $62(23,3)$ & \\
\hline 30 a 35 anos & $569(29,2)$ & $155(27,2)$ & & $105(32,7)$ & & $50(20,2)$ & \\
\hline Cor da pele & & & 0,085 & & 0,034 & & 0,964 \\
\hline Branco & $1.483(75,9)$ & $392(26,4)$ & & $251(30,8)$ & & $141(21,1)$ & \\
\hline Não branco & $470(24,1)$ & $144(30,6)$ & & $98(38,1)$ & & $46(21,6)$ & \\
\hline Anos de estudos" & & & 0,001 & & 0,000 & & 0,002 \\
\hline 0 a 7 anos & $258(13,2)$ & $113(43,8)$ & & $77(53,5)$ & & $36(31,6)$ & \\
\hline 8 a 10 anos & $339(17,4)$ & $108(31,9)$ & & $63(39,6)$ & & $45(25,0)$ & \\
\hline 11 ou mais anos & $1.352(69,4)$ & $314(23,2)$ & & $209(27,1)$ & & $105(18,0)$ & \\
\hline IEN1 ${ }^{\#}$ & & & 0,001 & & 0,001 & & 0,001 \\
\hline Baixa & $648(33,3)$ & $224(34,6)$ & & $160(40,4)$ & & $64(25,4)$ & \\
\hline Média & $655(33,7)$ & $183(27,9)$ & & $108(30,6)$ & & $75(24,8)$ & \\
\hline Alta & $641(33,0)$ & $125(19,5)$ & & $78(24,5)$ & & $47(14,6)$ & \\
\hline Situação conjugal ${ }^{\sharp}$ & & & 0,837 & & 0,799 & & 0,763 \\
\hline Solteiro & $1.230(63,1)$ & $335(27,2)$ & & $215(32,9)$ & & $120(20,8)$ & \\
\hline $\begin{array}{l}\text { Vive com } \\
\text { companheiro(a) }\end{array}$ & $720(36,9)$ & $200(27,8)$ & & $134(32,0)$ & & $66(21,9)$ & \\
\hline$I M C^{\#}$ & & & 0,506 & & 0,203 & & 0,416 \\
\hline Eutrófico & $860(48,3)$ & $235(27,3)$ & & $150(30,4)$ & & $85(23,3)$ & \\
\hline Sobrepeso & $565(31,7)$ & $149(26,4)$ & & $90(32,8)$ & & $59(20,3)$ & \\
\hline Obeso & $355(20,0)$ & $106(29,9)$ & & $81(37,2)$ & & $25(18,2)$ & \\
\hline Trabalho atual" & & & 0,307 & & 0,482 & & 0,480 \\
\hline Sim & $1.158(59,3)$ & $307(26,5)$ & & $147(33,8)$ & & $81(22,6)$ & \\
\hline Não & $794(40,7)$ & $228(28,7)$ & & $201(31,6)$ & & $106(20,3)$ & \\
\hline Doenças crônicas" & & & 0,001 & & 0,005 & & 0,002 \\
\hline Sim & $464(23,8)$ & $166(35,8)$ & & $235(30,0)$ & & $133(18,9)$ & \\
\hline Não & $1.486(76,2)$ & $368(24,8)$ & & $113(39,2)$ & & $53(30,1)$ & \\
\hline Tabagismo" & & & 0,001 & & 0,001 & & 0,001 \\
\hline Sim & $421(21,7)$ & $187(44,4)$ & & $121(50,8)$ & & $66(36,1)$ & \\
\hline Não & $1.521(78,3)$ & $345(22,7)$ & & $224(27,1)$ & & $121(17,5)$ & \\
\hline Álcool & & & 0,001 & & 0,001 & & 0,001 \\
\hline Sim & $186(9,56)$ & $77(41,2)$ & & $31(58,5)$ & & $45(33,8)$ & \\
\hline Não & $1.766(90,4)$ & $459(26,0)$ & & $318(31,2)$ & & $142(19,0)$ & \\
\hline Total & $1.953(100)$ & $536(27,4)$ & --. & $1.073(54,9)$ & -.- & $187(45,1)$ & --. \\
\hline
\end{tabular}

'Indicador Econômico Nacional - Brasil.

"Presença de missing.

idade ( $p=0,002)$, com menos anos de estudo ( $p<0,001)$, com menor tercil de renda ( $p<0,001)$, que relatou doença crônica ( $p<0,001)$, fumantes $(p<0,001)$ e abuso de álcool $(p=0,017)$.

A tabela 2 mostra as prevalências para cada quadro de transtorno de ansiedade e os fatores associados investigados no estudo.

\section{DISCUSSÃO}

Foi verificada alta prevalência de transtorno de ansiedade na população investigada: 27,4\% ( $n=536)$. Quando avaliados individualmente, os quadros mais prevalentes foram os de TAG e agorafobia. As variáveis sexo, anos de estudo, renda, doenças crônicas, tabagismo e abuso de álcool foram associadas a mais de três transtornos de ansiedade investigados. Molina et al. ${ }^{10}$, que investigaram a prevalência de comorbidades entre transtornos de humor e de ansiedade, encontraram agorafobia (12,3\%) e TAG (9,7\%) como diagnósticos mais prevalentes em sua amostra ${ }^{10}$, resultados menores do que os encontrados neste estudo, mas que corroboram os achados de prevalência.

Os problemas relacionados à saúde mental têm sido relacionados a múltiplos fatores sociais, culturais, econômicos e ambientais. Os contextos social, educativo e de trabalho e o 
Tabela 2. Quadros de transtorno de ansiedade e fatores associados investigados

\begin{tabular}{|c|c|c|c|c|c|c|c|c|c|c|c|c|}
\hline Variáveis & Agorafobia & $p$ & $\begin{array}{l}\text { Transtorno } \\
\text { de Pânico }\end{array}$ & $p$ & TOC 1 & $p$ & Fobia Social & $p$ & TEPT $^{2}$ & $p$ & $\mathrm{TAG}^{3}$ & $p$ \\
\hline Sexo & & 0,001 & & 0,838 & & 0,009 & & 0,001 & & 0,004 & & 0,001 \\
\hline Feminino & $222(20,7)$ & & $53(68,8)$ & & $57(5,3)$ & & $79(7,4)$ & & $43(4,0)$ & & $200(18,7)$ & \\
\hline Masculino & $128(14,6)$ & & $18(64,3)$ & & $25(2,8)$ & & $26(3,0)$ & & $15(1,7)$ & & $78(8,9)$ & \\
\hline Idade & & $0,763^{*}$ & & $0,757^{*}$ & & 0,587 & & 0,010 & & $0,540^{*}$ & & $0,002^{*}$ \\
\hline 18 a 23 anos & $141(18,5)$ & & $18(72,0)$ & & $34(4,5)$ & & $36(4,7)$ & & $20(2,6)$ & & $84(11,0)$ & \\
\hline 24 a 29 anos & $107(17,2)$ & & $24(64,9)$ & & $22(3,5)$ & & $25(4,0)$ & & $20(3,2)$ & & $99(16,0)$ & \\
\hline 30 a 35 anos & $102(18,0)$ & & $29(67,4)$ & & $26(4,6)$ & & $44(7,8)$ & & $18(3,2)$ & & $95(16,8)$ & \\
\hline Cor da pele & & 0,147 & & 0,921 & & 0,205 & & 0,008 & & 0,415 & & 0,240 \\
\hline Branco & $255(17,2)$ & & $50(66,7)$ & & $57(3,9)$ & & $68(4,6)$ & & $41(2,8)$ & & $203(13,7)$ & \\
\hline Não branco & $95(20,3)$ & & $21(70,0)$ & & $25(5,4)$ & & $37(7,9)$ & & $17(3,6)$ & & $75(16,0)$ & \\
\hline Anos de estudo & & 0,001 & & 0,778 & & 0,004 & & 0,000 & & 0,001 & & 0,001 \\
\hline 0 a 7 anos & $83(32,2)$ & & $21(72,4)$ & & $20(7,8)$ & & $35(13,6)$ & & $19(7,4)$ & & $60(23,3)$ & \\
\hline 8 a 10 anos & $78(23,1)$ & & $11(68,8)$ & & $17(5,0)$ & & $21(6,2)$ & & $15(4,4)$ & & $46(13,5)$ & \\
\hline 11 anos ou mais & $188(13,9)$ & & $39(65,0)$ & & $45(3,3)$ & & $49(3,6)$ & & $24(1,8)$ & & $172(12,8)$ & \\
\hline $\mathrm{IEN}^{4}$ & & 0,001 & & $0,032^{*}$ & & $0,001 *$ & & $0,001^{*}$ & & $0,001^{*}$ & & $0,001^{*}$ \\
\hline Baixa & $146(22,5)$ & & $36(78,3)$ & & $41(6,4)$ & & $51(7,9)$ & & $34(5,3)$ & & $123(19,0)$ & \\
\hline Média & $121(18,5)$ & & $21(61,8)$ & & $26(4,0)$ & & $37(5,7)$ & & $17(2,6)$ & & $93(14,2)$ & \\
\hline Alta & $80(12,5)$ & & $13(54,2)$ & & $14(2,2)$ & & $16(2,5)$ & & $5(0,8)$ & & $59(9,2)$ & \\
\hline Situação conjugal & & 0,866 & & 0,129 & & 0,697 & & 0,499 & & 0,425 & & 0,708 \\
\hline Solteiro & $222(18,1)$ & & $42(75,0)$ & & $54(4,4)$ & & $70(5,7)$ & & $40(3,3)$ & & $172(14,0)$ & \\
\hline $\begin{array}{l}\text { Vive com } \\
\text { companheiro(a) }\end{array}$ & $127(17,7)$ & & $29(59,2)$ & & $28(3,9)$ & & $35(4,9)$ & & $18(2,5)$ & & $106(14,7)$ & \\
\hline IMC & & 0,585 & & $0,163^{*}$ & & $0,631 *$ & & 0,125 & & 0,106 & & $0,212^{*}$ \\
\hline Eutrófico & $155(18,1)$ & & $33(76,7)$ & & $40(4,7)$ & & $53(6,2)$ & & $31(3,6)$ & & $116(13,5)$ & \\
\hline Sobrepeso & $96(17,0)$ & & $15(60,0)$ & & $22(3,9)$ & & $22(3,9)$ & & $10(1,8)$ & & $76(13,5)$ & \\
\hline Obeso & $70(19,7)$ & & $18(62,1)$ & & $15(4,2)$ & & $23(6,5)$ & & $13(3,7)$ & & $59(16,7)$ & \\
\hline Trabalho atual & & 0,344 & & 1,000 & & 0,344 & & 0,070 & & 0,600 & & 0,459 \\
\hline Sim & $199(17,2)$ & & $38(67,9)$ & & $44(3,8)$ & & $53(4,6)$ & & $32(2,8)$ & & $159(13,7)$ & \\
\hline Não & $150(19,0)$ & & $33(67,3)$ & & $38(4,8)$ & & $52(6,6)$ & & $26(3,3)$ & & $119(15,0)$ & \\
\hline Doenças crônicas & & 0,001 & & 0,662 & & 0,008 & & 0,001 & & 0,002 & & 0,001 \\
\hline Sim & $115(24,8)$ & & $34(70,8)$ & & $30(6,5)$ & & $46(9,9)$ & & $24(5,2)$ & & $104(22,5)$ & \\
\hline Não & $233(15,7)$ & & $37(64,9)$ & & $52(3,5)$ & & $59(4,0)$ & & $34(2,3)$ & & $174(11,7)$ & \\
\hline Tabagismo & & 0,001 & & 0,014 & & 0,001 & & 0,001 & & 0,000 & & 0,001 \\
\hline Sim & $127(30,2)$ & & $38(80,9)$ & & $35(8,4)$ & & $39(9,3)$ & & $28(6,7)$ & & $103(24,5)$ & \\
\hline Não & $219(14,4)$ & & $32(56,1)$ & & $47(3,1)$ & & $66(4,3)$ & & $30(2,0)$ & & $173(11,4)$ & \\
\hline Abuso de álcool & & 0,001 & & 0,949 & & 0,002 & & 0,460 & & 0,002 & & 0,017 \\
\hline Sim & $51(27,3)$ & & $15(68,2)$ & & $16(8,6)$ & & $13(7,0)$ & & $13(7,0)$ & & $38(20,3)$ & \\
\hline Não & $299(17,0)$ & & $56(67,5)$ & & $66(3,8)$ & & $92(5,2)$ & & $45(2,6)$ & & $240(13,6)$ & \\
\hline
\end{tabular}

${ }^{1}$ Transtorno obsessivo-compulsivo.

${ }^{2}$ Transtorno de estresse pós-traumático.

${ }^{3}$ Transtorno de ansiedade generalizada.

${ }^{4}$ Indicador Econômico Nacional - Brasil.

* Coeficiente de linearidade. 
acesso aos serviços de saúde podem ser identificados como estressores psicossociais e ambientais. As altas prevalências encontradas para agorafobia, TAG e fobia social podem estar relacionadas ao grupo social em que estavam inseridos os indivíduos estudados, a pouco apoio social e a dificuldades de adaptação ao meio, ou ainda à existência de problemas no trabalho ou ambiente educacional, com a experimentação de situações de desaprovação e rechaço ${ }^{11}$. Por fim, problemas econômicos também podem estar relacionados à alta prevalência desses quadros, em virtude da dificuldade de cumprir com a responsabilidade de efetuar pagamentos, comprar bens necessários e manter hábitos.

No presente estudo, as mulheres foram mais acometidas pelos transtornos de ansiedade, comparadas aos homens em todos os quadros investigados. Segundo Kinrys e Wygant, as mulheres têm maior risco de desenvolver transtornos de ansiedade ao longo da vida'12. É possível que sejam mais acometidas por quadros de ansiedade em razão da pressão social que recebem, da jornada de trabalho e da renda inferior. Isso faz com que muitas tenham que trabaIhar mais para cumprir com pagamentos e manutenção da família ${ }^{13}$. Outra possível justificativa pode estar relacionada à exposição à violência que a mulher vem enfrentando cotidianamente, o que pode deixá-la em constante sensação de medo, angústia e ansiedade ${ }^{14}$. No entanto, ainda que as muIheres apresentem maior gravidade nos sintomas e evolução crônica nos transtornos de ansiedade ${ }^{12}$, é preciso salientar que, independentemente do sexo, os transtornos de ansiedade podem causar grande prejuízo funcional na vida dos indivíduos e consequências graves, como a dificuldade de arranjar emprego, a de conviver em grupos e de participar de atividades de lazer.

De modo geral, existe pouca pesquisa no Brasil que discuta a relação da raça/cor da pele com os transtornos emocionais, principalmente porque há dificuldade em classificar raça em um país miscigenado e a oposição entre classe social e raça. A raça/cor da pele pode interferir nas oportunidades educacionais, financeiras e sociais, influenciando a posição socioeconômica no contexto atual de quem vive no Brasil. E as características socioeconômicas apresentam maior relação com os transtornos de ansiedade do que a própria raça do indivíduo ${ }^{15}$.

Dados publicados em 2017 pela da Organização Mundial de Saúde (OMS) mostram que a prevalência mundial do transtorno de ansiedade é de aproximadamente 3,6\%, alcançando maiores proporções no continente americano quando relacionado ao sexo; para as mulheres, atinge $7,7 \%$ e para os homens, 3,6\% ${ }^{16}$. Esses dados estão em conformidade com o presente estudo, mostrando que o sexo feminino é o mais acometido. $O$ transtorno de ansiedade foi bastante prevalente em todas as faixas etárias estudadas. Entretanto, não apresentou diferença significativa quanto à idade. O Relatório de Saúde Mental da ONU, em 2001, já apontava o quanto o transtorno de ansiedade era comum e incapacitante em todas as idades ${ }^{6}$.

O Brasil, onde o transtorno de ansiedade está presente em 9,3\% da população geral, se destaca, possuindo o maior número de casos de ansiedade entre todos os países do mundo ${ }^{16}$. A prevalência encontrada neste estudo, de 27,4\%, supera os dados estimados para o Brasil pela OMS, porém os participantes da pesquisa apresentam uma idade mais vulnerável para a ansiedade, por estarem em busca do engajamento no mercado de trabalho, reconhecimento profissional e, muitas vezes, passando pela experiência da maternidade e formação de família, visto que essa amostra está compreendida entre 18 e 35 anos de idade. Em outro estudo populacional com participantes de 18 a 24 anos, foram diagnosticados $12,3 \%$ dos participantes com transtorno de ansiedade ${ }^{17}$. Quando analisados, estudos com crianças e adolescentes mostram que a prevalência de ansiedade na vida adulta pode ser um reflexo originado desde a infância e juventude; dados de uma revisão sistemática com crianças e jovens revelaram uma variação nas taxas de transtornos de ansiedade entre 3,3\% e 32,3\% ${ }^{18}$. Assim como esses transtornos podem prejudicar o desenvolvimento das crianças e adolescentes, podem também levar a consequências como prejuízo funcional e social, afetando a vida de adultos.

O grupo com menos anos de estudo foi o que apresentou maior prevalência de transtornos de ansiedade $(p<0,001)$. Os anos de estudo também apresentaram relação com a prevalência dos quadros individuais de transtornos de ansiedade (exceto para o transtorno de pânico, que não teve relação estatisticamente significativa). Quanto menos anos de estudo, maior a prevalência de TAG, fobia social, TOC, TEPT e agorafobia. Na literatura, é encontrada relação da maior prevalência de transtornos de ansiedade e menor escolaridade ${ }^{19}$, e uma das hipóteses para esse achado pode estar relacionada às dificuldades que os jovens enfrentam no ambiente escolar e acadêmico, como as preocupações com o desempenho ou déficits de aprendizagem, e os transtornos de ansiedade podem limitar os indivíduos na tentativa de atingirem maior rendimento escolar ${ }^{11}$. A baixa escolaridade ainda pode estar relacionada a menor renda e piores oportunidades de trabaIho. Dado semelhante ao do presente estudo foi encontrado por Souza ${ }^{20}$ e Torres et al..21, que associaram a renda mais baixa à presença de $\mathrm{TAG}^{20}$ e TOC ${ }^{21}$. A exposição a condições socioeconômicas mais desfavorecidas e fatores relacionados (violência, dificuldade de acesso aos serviços de saúde, desemprego, má distribuição econômica, entre outros) pode ser potencializadora dos quadros de ansiedade, facilitando tanto o surgimento como o agravo daqueles já existentes.

Mais de 50\% da amostra estava acima do peso, e 20\% desses eram obesos. Apesar de a obesidade não estar classificada como um transtorno psiquiátrico ${ }^{22}$, obesos apresentam maior incidência de psicopatologias ${ }^{23}$. Entretanto, as complicações psiquiátricas são muito difíceis de ser ca- 
racterizadas nos casos de obesidade ${ }^{24}$, ou seja, a obesidade pode gerar quadros psiquiátricos, mas também pode ser consequência deles. Entre os transtornos mais relacionados com o excesso de peso, estão agorafobia, TAG, transtorno de pânico e TEPT 25 .

Segundo o IBGE, a região Sul tem o maior percentual de fumantes do país, alcançando 19\% ${ }^{26}$. Apesar da redução da prevalência nos últimos anos, o número de fumantes ainda é alto. Os malefícios do hábito são conhecidos, e o tabagismo caracteriza-se como a principal causa de morte evitável no mundo ${ }^{27}$. O presente estudo apresentou a relação entre usar tabaco e ansiedade $(p<0,001)$, e essa relação permaneceu significava mesmo quando estratificada pelo sexo $(p<0,001)$.

Houve também relação significativa quando os transtornos foram avaliados de forma individual. Ou seja, os fumantes também apresentaram maior prevalência de agorafobia, TAG, fobia social, TOC, transtorno de pânico e TEPT. Segundo Valença et al.28, estudos vêm demonstrando associação positiva entre tabagismo e transtornos psiquiátricos.

Estudos de revisão tanto com animais como em humanos relacionam a nicotina com a ansiedade e identificaram uma série de genes e receptores nicotínicos de acetilcolina envolvidos nesse contexto, podendo ser ansiolítico pelas vias GABAérgicas ou ansiogênicos por vias serotoninérgicas $^{29}$. Rondina et al. ${ }^{30}$ colocam que a natureza da relação entre tabagismo e transtorno de ansiedade varia de acordo com o tipo de diagnóstico, e a prevalência de tabagismo em indivíduos com TOC seria menor por causa dos sistemas serotonérgico e colinérgico. Ao mesmo tempo, o tabagismo poderia ser um fator de risco para o aparecimento do transtorno de pânico. Todavia, a relação entre ansiedade e tabagismo é muito variável. Comparados à população geral, indivíduos com ansiedade têm duas vezes mais propensão a fumar, do mesmo modo que esses transtornos são mais comuns em indivíduos fumantes ${ }^{31}$. O uso de cigarro é compreendido pelos indivíduos como um hábito que diminui a ansiedade, outras vezes a cessação, a cronicidade e doses altas do cigarro podem ser entendidas como antigênico. Em algumas pessoas, a nicotina realmente reduz os sintomas de ansiedade quando em doses baixas e pode ser interpretada como ansiolítica, porém pode perpetuar o hábito de fumar e a dependência ${ }^{32,33}$.

No que diz respeito ao uso de álcool e ao transtorno de ansiedade, existe associação. Quando esses dados foram estratificados por sexo, 58,5\% $(n=31)$ das mulheres usuárias de álcool apresentaram ansiedade ( $p<0,001)$, bem como 33,8\% $(n=45)$ dos homens $(p<0,001)$. Nos quadros individuais de ansiedade, 27,3\% ( $n=51)$ dos usuários de álcool apresentaram agorafobia $(p=0,001), 8,6 \%(n=16)$, TOC $(p=0,002)$, $7,0 \%(n=13)$, TEPT $(P=0,002)$ e 20,3\% ( $n=38)$, TAG $(p=0,017)$.

Costa et al. encontraram associação positiva entre ansiedade e uso de álcool em adultos, nos quais o hábito é realizado como automedicação, dependendo do tipo de transtorno ${ }^{34}$. Em um estudo com adolescentes, a ansiedade social foi um fator de risco para o uso de álcoo ${ }^{35}$. Indivíduos alcoolistas apresentam quadros significativos de ansiedade, como transtornos de pânico, fóbicos e de ansiedade generalizada ${ }^{36}$. O TEPT também foi associado ao uso de álcool, drogas e nicotina no estudo de Breslau ${ }^{37}$; nesses casos os indivíduos também utilizam o álcool para aliviar sintomas de ansiedade. O TEPT ainda aparece como comorbidade em alcoolistas e como o transtorno mais frequente em indivíduos que usavam ou eram dependentes de álcool ${ }^{38}$. Já o estudo de Souza sobre o TAG em jovens entre 18 e 24 anos e fatores associados encontrou uso de álcool em 81,7\% da amostra ${ }^{20}$. A relação do uso do álcool e ansiedade é complexa, podendo agravar quadros e levar à dependência. Muitos indivíduos utilizam álcool para aliviar sintomas de ansiedade e tornarem-se mais confiantes e independentes em suas atividades rotineiras e que exigem contato social. Cabe ressaltar que o presente estudo considerou a associação entre uso abusivo de álcool e transtornos de ansiedade.

A presença de doenças crônicas (de diferentes ordens), a iminência de agravos e a dificuldade de acesso aos serviços de saúde podem ser geradoras de ansiedade. Esse estudo encontrou essa associação, ou seja, o grupo que relatou ter alguma doença crônica apresentou maior prevalência de ansiedade $(p<0,001)$. E quando os transtornos foram avaliados individualmente, o grupo com doenças crônicas apresentou maior prevalência em todos os quadros. Alguns transtornos psiquiátricos são comórbidos, como os casos de TEPT, que podem ser precedidos por outros quadros de saúde mental e até levar ao suicídio. A relação inversa também é possível: transtornos de ansiedade já existentes podem aumentar a exposição a traumas e, consequentemente, levar ao TEPT ${ }^{39}$. O TAG também tem altas taxas de comorbidade. A presença desse transtorno aumenta em 20 vezes o risco de o indivíduo apresentar transtorno de pânico ${ }^{6}$. Também no estudo de Souza ${ }^{20}$, comorbidades ao TAG foram encontradas na seguinte ordem: transtorno de pânico (9,2\%), agorafobia (37,5\%), fobia social (16,4\%), TOC (14,5\%) e TEPT (9,9\%). Assim, é importante salientar que os quadros aqui estudados podem estar relacionados entre si e até mesmo sobrepor diagnósticos, o que aumentaria a prevalência desses transtornos.

Contudo, o delineamento transversal do estudo impede uma explicação profunda do fenômeno existente na relação entre transtornos de ansiedade e os fatores aqui analisados. O estudo apenas evidencia que, de forma independente da relação com as características investigadas, tal relação existe. Além disso, o delineamento impossibilita a identificação de causalidade entre essas variáveis. Outra limitação que deve ser levada em consideração, apesar da adequação psicométrica à entrevista MINI, é que o processo de avaliação pode ter sido influenciado pela brevidade da realização do instrumento, falta de motivação do respondente, entre outras características que divergem da situação ideal do referido 
processo. Contudo, a originalidade e o rigor metodológico empregados em sua execução dão credibilidade aos achados aqui expostos.

O presente estudo identificou que os indivíduos do sexo feminino, com menos anos de estudo, baixo nível socioeconômico, histórico de doenças crônicas, usuários de tabaco e abusadores de álcool foram os que apresentaram maior probabilidade de apresentar transtornos de ansiedade.

Tais congruências sinalizam a necessidade de maior acurácia nas intervenções em saúde mental, em especial no serviço de saúde pública, local de maior procura de indivíduos com os fatores acima destacados. Da mesma forma, o sexo feminino, baixa condição socioeconômica e sustâncias lícitas podem ser importantes características a serem levadas em consideração pelo profissional da saúde, para que estratégias efetivas sejam implementadas levando em consideração o contexto real.

\section{CONTRIBUIÇÕES INDIVIDUAIS}

Todos os autores contribuíram significativamente na redação do artigo, análise e interpretação dos dados, bem como na aprovação da sua versão final a ser publicada.

\section{CONFLITO DE INTERESSES}

Não há conflito de interesses a serem declarados.

\section{AGRADECIMENTO}

O estudo foi apoiado pela Coordenação de Aperfeiçoamento de Pessoal de Nível Superior (Capes), Conselho Nacional de Desenvolvimento Científico e Tecnológico (CNPQ) e Fundação de Amparo à Pesquisa do Estado do Rio Grande do Sul (Fapergs), Brasil.

\section{REFERÊNCIAS}

1. Oreskovic S. Breaking down the Silo Mentality in Global Mental Health: The New Role for the Schools of Public Health. Psychiatr Danub. 2016;28(4):318-20.

2. Baxter AJ, Scott KM, Vos T, Whiteford HA. Global prevalence of anxiety disorders: a systematic review and meta-regression. Psychol Med. 2013;43(5):897-910.

3. Associação de Psiquiatria Americana. Manual Diagnóstico e Estatístico de Transtornos Mentais. Rev: DSM-IV-TRTM. Trad.: Cláudia Dornelles. Porto Alegre: Artmed; 2002.

4. Castillo ARG, Recondo R, Asbahr FR, Manfro GG. Transtornos de ansiedade. Rev Bras Psiquiat. 2000;22(2):20-3

5. Machado MB, Ignácio ZM, Jornada LK, Réus GZ, Abelaira HM, Arent CO, et al. Prevalência de transtornos ansiosos e algumas comorbidades em idosos: um estudo de base populacional. J Bras Psiquiatr. 2016;65(1):28-35.
6. Sayers J. The world health report 2001 - Mental health: new understanding, new hope. Bull World Health Organ. 2001;79(11):1085.

7. Masur J, Monteiro MG. Validation of the "CAGE" alcoholism screening test in a Brazilian psychiatric inpatient hospital setting. Braz J Med Biol Res. 1983;16(3):215-8.

8. Brasil. Ministério da Saúde. Orientações para a coleta e análise de dados antropométricos em serviços de saúde: Norma Técnica do Sistema de Vigilância Alimentar e Nutricional Sisvan. Braślila: Ministério da Saúde; 2011

9. Amorim P. Mini International Neuropsychiatric Interview (MINI): validação de entrevista breve para diagnóstico de transtornos mentais. Rev Bras Psiquiatr. 2000;22(3):106-15.

10. Molina MRAL, Spessato B, Jansen K, Pinheiro R, Silva R, Souza LDM. Prevalence of comorbidities between mood and anxiety disorders: associated factors in a population sample of young adults in southern Brazil. Cad Saúde Pública. 2014;30(11):2413-22.

11. Londoño NH, Juárez F, Palacio J, Muñiz O, Agudelo D, Marín CA, et al. Factores de riesgo psicosociales y ambientales asociados a trastornos mentales. Suma Psicol. 2010;17(1):5968.

12. Kinrys $G$, Wygant LE. Anxiety disorders in women: does gender matter to treatment? Rev Bras Psiquiatr. 2005;27(Suppl 2):43-50

13. Fiorin $P C$, Oliveira CT, Dias ACG. Percepçoes de mulheres sobre a relação entre trabalho e maternidade. Rev Bras Orientac Prof. 2014;15(1):25-35.

14. Waiselfisz JJ. Mapa da Violência 2012 - Atualização: Homicídios de mulheres no Brasil. 2012. Disponível em: https://www.mapadaviolencia.org.br/pdf2012/MapaViolencia2012_atual_mulheres.pdf. Acesso em: 2 fev. 2019.

15. Smolen JR, Araújo EM. Raça/cor da pele e transtornos mentais no Brasil: uma revisão sistemática. Ciênc Saúde Coletiva. 2017:22(12):4021-30.

16. World Health Organization (WHO). Depression and other common mental disorders: global health estimates. Genebra: WHO; 2017.

17. Mondin TC, Konradt CE, Cardoso TA, Quevedo LA, Jansen K, Mattos LD, et al. Anxiety disorders in young people: a population-based study. Braz J Psychiatry. 2013;35(4):347-52.

18. Thiengo DL, Cavalcante MT, Lovisi GM. Prevalência de transtornos mentais entre crianças e adolescentes e fatores associados: uma revisão sistemática. J Bras Psiquiatr. 2014;63(4):360-72

19. Andrade LH, Wang YP, Andreoni S, Silveira CM, Alexandrino-Silva C, Siu ER, et al. Mental disorders in megacities: findings from the Sao Paulo megacity mental health survey, Brazil. Plos One. 2012;7(2):e31879.

20. Souza LDM. Transtorno de ansiedade generalizada em jovens de 18 a 24 anos: prevalência, fatores associados, comorbidades e impacto na qualidade de vida [tese]. Porto Alegre: Pontifícia Universidade Católica do Rio Grande do Sul; 2010.

21. Torres AR, Prince MJ, Bebbington PE, Bhugra D, Brugha TS, Farrell M, et al. Obsessivecompulsive disorder: prevalence, comorbidity, impact, and help-seeking in the British National Psychiatric Morbidity Survey of 2000. Am J Psychiatry. 2006;163(11):1978-85.

22. Costa FS, Bandeira DR, Trentini CM, Brilmann M, Friedman R, Nunes MA. Considerações acerca da avaliação psicológica das comorbidades psiquiátricas em obesos. Psicol Estud. 2009;14(2):287-93

23. Becker E, Margraf J, Türke V, Soeder U, Neumer S. Obesity and mental illness in a representative sample of young women. Int J Obes Relat Metab Disord. 2001;25 Suppl 1:S5-9.

24. Nogueira TFD, Zambon MP. Reasons for non-adherence to obesity treatment in children and adolescents. Rev Paul Pediatr. 2013:31(3):338-43.

25. Dobrow IJ, Kamenetz C, Devlin MJ. Aspectos psiquiátricos da obesidade. Rev Bras Psiquiatr. 2002:24(Supl 3):63-7.

26. Instituto Brasileiro de Geografia e Estatística (2009). Pesquisa Especial do Tabagismo 2008. Pesquisa Nacional por Amostra de Domićlio. IBGE; 2009.

27. Sattler AC, Cade NV. Prevalência da abstinência ao tabaco de pacientes tratados em unidades de saúde e fatores relacionados. Ciênc Saúde Coletiva. 2013:18(1):253-64.

28. Valença AM, Nardi AE, Nascimento I, Mezzasalma MA, Lopes FL, Zin W. Transtorno de pânico e tabagismo. Rev Bras Psiquiatr. 2001;23(4):229-32.

29. Mineur YS, Picciotto MR. Genetics of nicotinic acetylcholine receptors: relevance to nicotine addiction. Biochem Pharmacol. 2008;75(1):323-33.

30. Rondina RC, Gorayeb R, Botelho C. Características psicológicas associadas ao comportamento de fumar tabaco. J Bras Pneumol. 2007;33(5):592-601. 
31. Pawlina MMC, Rondina RC, Espinosa MM, Botelho C. Depression, anxiety, stress, and motivation over the course of smoking cessation treatment. J Bras Pneumol. 2015;41(5):433-9.

32. Pawlina MMC, Rondina RC, Espinosa MM, Botelho C. Ansiedade e baixo nível motivacional associados ao fracasso na cessação do tabagismo. J Bras Psiquiatr. 2014;63(2):113-20

33. Cheeta S, Irvine EE, Kenny PJ, File SE. The dorsal raphe nucleus is a crucial structure mediating nicotine's anxiolytic effects and the development of tolerance and withdrawal responses. Psychopharmacology. 2001;155(1):78-85.

34. Costa MA, Salum Junior GA, Isolan LR, Acosta JR, Jarros RB, Blaya C, et al. Association between anxiety symptoms and problematic alcohol use in adolescents. Trends in Psychiatry and Psychother. 2013;35(2):106-10
35. Vilela L, Macho P, Almeida G. Consumo de álcool em adolescentes e psicopatologia associada. Toxicodependências. 2011;17(1):43-52.

36. Peuker AC, Rosemberg R, Cunha SM, Araújo LB. Fatores associados ao abuso de drogas em uma população clínica. Paidéia (Ribeirão Preto). 2010;20(46):165-73.

37. Breslau N. Epidemiologic studies of trauma, posttraumatic stress disorder, and other psychiatric disorders. Los Angeles, CA: Sage Publications; 2002.

38. Pulcherio G, Vernetti C, Strey MN, Faller S. Post-traumatic stress disorder and alcoholism. Arch Clin Psychiatry. 2008;35(4):154-8

39. Quarantini LC, Netto LR, Andrade-Nascimento M, Almeida AG, Sampaio AS, Miranda-Scippa A, et al. Comorbid mood and anxiety disorders in victims of violence with posttraumatic stress disorder. Rev. Bras. Psiquiatr. 2009;31(Supl 2):66-76. 